\title{
Clinical implications of TPO and AOX1 in pediatric papillary thyroid carcinoma
}

\author{
Yongbo Yu ${ }^{1 \#}$, Shengcai Wang ${ }^{2 \#}$, Xuexi Zhang ${ }^{2}$, Shuai $\mathrm{Xu}^{3}$, Yanzhen $\mathrm{Li}^{2}$, Qiaoyin Liu ${ }^{2}$, Yeran Yang ${ }^{1}$, \\ Nian Sun ${ }^{2}$, Yuanhu Liu ${ }^{2}$, Jie Zhang ${ }^{2}$, Yongli Guo ${ }^{1}$, Xin Ni, \\ ${ }^{1}$ Beijing Key Laboratory for Pediatric Diseases of Otolaryngology, Head and Neck Surgery, Beijing Pediatric Research Institute, Beijing Children's \\ Hospital, Capital Medical University, National Center for Children's Health (NCCH), Beijing, China; ${ }^{2}$ Department of Otolaryngology, Head and \\ Neck Surgery, Beijing Children's Hospital, Capital Medical University, National Center for Children's Health (NCCH), Beijing, China; ${ }^{3}$ Department \\ of Urology, Beijing Children's Hospital, Capital Medical University, National Center for Children's Health (NCCH), Beijing, China \\ Contributions: (I) Conception and design: Y Yu, S Wang, X Ni; (II) Administrative support: S Wang, Y Guo; (III) Provision of study materials or \\ patients: X Zhang, S Xu, Y Li, Q Liu; (IV) Collection and assembly of data: Y Yang, N Sun; (V) Data analysis and interpretation: Y Liu, J Zhang; (VI) \\ Manuscript writing: All authors; (VII) Final approval of manuscript: All authors. \\ \#These authors contributed equally to this work. \\ Correspondence to: Xin Ni. Department of Otolaryngology, Head and Neck Surgery, Beijing Children's Hospital, Capital Medical University, National \\ Center for Children's Health (NCCH), NO.56, Nanlishi Road, Xicheng District, Beijing 100045, China. Email: nixin@bch.com.cn; Yongli Guo. \\ Beijing Key Laboratory for Pediatric Diseases of Otolaryngology, Head and Neck Surgery, Beijing Pediatric Research Institute, Beijing Children's \\ Hospital, Capital Medical University, National Center for Children's Health (NCCH), NO.56, Nanlishi Road, Xicheng District, Beijing 100045, \\ China. Email: guoyongli@bch.com.cn.
}

Background: Thyroid carcinoma is a common pediatric head and neck cancer, of which papillary thyroid cancer (PTC) is the most common type. Previously, we found that thyroid peroxidase (TPO) and aldehyde oxidase $1(A O X 1)$ were differentially expressed in PTC. This study explored the clinical importance of TPO and $A O X 1$ in the diagnosis and prognosis of PTC in children.

Methods: Both TPO and AOX1 expression in PTC were analyzed using datasets from Gene Expression Omnibus (GEO). TPO and AOX1 protein levels in plasma from patients with PTC and non-tumor controls were detected via enzyme-linked immunosorbent assay (ELISA). The diagnostic accuracy of TPO and AOX1 was assessed using receiver operating characteristic (ROC) curve analysis. The association between gene expression levels and patient survival was explored using the Kaplan-Meier plotter online database.

Results: The results revealed that TPO and $A O X 1$ expression was significantly downregulated in four independent datasets (GSE33630, GSE27155, GSE3678, and GSE3467). TPO and AOX1 protein levels in blood plasma were significantly decreased in patients with PTC. Quantitative analysis demonstrated that TPO and AOX1 levels in plasma had satisfactory predictive performance and the ability to discriminate PTC from healthy samples. Prognostic analysis demonstrated that low levels of TPO and AOX1 were markedly associated with poor survival in patients with PTC.

Conclusions: In summary, these results implied that TPO and AOX1 could serve as novel biomarkers for the diagnosis and prognosis of pediatric PTC.

Keywords: Pediatrics; papillary thyroid carcinoma; thyroid peroxidase; aldehyde oxidase

Submitted Sep 18, 2020. Accepted for publication Feb 05, 2021.

doi: $10.21037 /$ tp-20-301

View this article at: http://dx.doi.org/10.21037/tp-20-301 


\section{Introduction}

Global cancer statistics revealed that 567,000 cases of thyroid cancer occur globally each year, ranking ninth among malignancies (1). Papillary thyroid cancer (PTC) is the most common type of thyroid cancer, accounting for at least $90 \%$ of all childhood cases. The incidence of PTC is increasing in many countries, including the USA, the UK, Denmark, and China (2-5), and the incidence rates increased more rapidly from 2006 to 2013 than from 1973 to 2006 (6). Because the 5-year survival of PTC usually exceeds $95 \%$ (7), it is considered a "curable" cancer. Occasionally, this malignancy can dedifferentiate into aggressive thyroid cancers, and more than $25 \%$ of patients with PTC experience recurrence during long-term followup (8). It is well known that the BRAF (V600E) mutation, $R A S$ mutation, and RET/PTC rearrangement are common gene alterations in PTC (9). In addition to gene mutations, RNA biomarkers and proteome-based biomarkers were recently identified, and it is hoped that these biomarkers can discriminate this cancer from non-cancer (8). However, the etiology and molecular biology of PTC remain clear. Previously, we found that both thyroid peroxidase (TPO) and aldehyde oxidase 1 (AOX1) were downregulated in PTC $(10,11)$. However, further experimental verification and studies of the clinical importance of TPO and $A O X 1$ in PTC must be conducted. Careful assessment of genetic features is considered useful for predicting clinical outcomes and planning treatment.

As a key enzyme involved in thyroid hormone synthesis, TPO is essential for thyroid function. Previously, peroxidase activity was detected at lower levels in thyroid carcinoma than in normal thyroid glands (12). TPO gene mutation and expression changes are also involved in thyroid cancer. A case-control study reported that a TPO variant is associated with thyroid carcinoma (13). Our previous study and other integrated analyses demonstrated that TPO was significantly downregulated in PTC $(10,14)$. Pathologically, immunohistochemical staining verified that TPO can distinguish PTC from benign thyroid lesions (15). However, TPO protein levels in blood, which might be a promising non-invasive index, have not been explored to date.

AOX1 is well described as a xenobiotic-metabolizing enzyme that plays roles in the metabolism of nitrogenous heterocyclic compounds and regulation of reactive oxygen species (ROS) and lipid metabolism $(16,17)$. A proteomic analysis found that $A O X 1$ expression was high in normal tissues but completely lacking in hepatocellular and pancreatic cancer $(16,18)$. Moreover, reduced $A O X 1$ expression is correlated with the hepatocellular tumor stage and metastatic/regional lymph node states (16). However, the association between $A O X 1$ expression and PTC pathogenesis is unclear.

Therefore, we measured the protein content of TPO and AOX1 in plasma from children with PTC. Diagnostic analysis was performed to determine whether TPO and AOX1 in plasma are promising biomarkers for PTC diagnosis. Using an online database, survival analysis was conducted to evaluate their clinical importance for PTC prognosis.

We present the following article in accordance with the REMARK reporting checklist (available at http://dx.doi. org/10.21037/tp-20-301).

\section{Methods}

\section{Blood samples and clinical information}

This study follows the Helsinki Declaration (as revised in 2013) and was approved by the Ethics Committee in Beijing Children's Hospital, Capital Medical University (2020-k-168). In total, data for 64 patients diagnosed with PTC (February 2012 to May 2019) were collected from the medical record system, and 40 blood samples were available in our Biobank of Clinical Resources. Forty cancer-free blood samples (physical examination) matched for age and gender served as the control group. The TNM pretreatment staging classification was used for the clinical staging of PTC. Informed consent was provided by each patient and/or his/her legal guardians before samples were collected and stored in the biobank.

\section{Public data preprocessing}

To verify the expression of TPO and AOX1, several Gene Expression Omnibus (GEO) datasets were analyzed. Series matrix files of GSE33630 (49 PTC, 45 normal), GSE27155 (51 PTC, 4 normal), GSE3678 (7 PTC, 7 normal), and GSE3467 (9 PTC, 9 normal) were downloaded. Normalized values of both TPO and $A O X 1$ were then selected to verify their expression in PTC and normal thyroid tissue.

\section{TPO and AOX1 detection}

According to the standard operating procedure in the biobank, venous blood samples were taken from each patient 
at the time of diagnosis. Blood samples were collected in EDTA anticoagulant and centrifuged at 3,000 rpm for $5 \mathrm{~min}$, and the supernatant was stored frozen at $-80{ }^{\circ} \mathrm{C}$ until analysis. The concentrations of TPO and AOX1 in plasma were measured using enzyme-linked immunosorbent assay (ELISA) kits (TPO, Cusabio, USA; AOX1, Abbexa, $\mathrm{UK}$ ) according to the manufacturer's instructions.

\section{Diagnostic testing}

Receiver operating characteristic (ROC) curves were used to evaluate classifiers in clinical diagnosis. To assess the predictive accuracy of TPO and AOX1 levels in pediatric PTC, ROC curves were generated using the TPO and AOX1 concentrations in plasma from pediatric patients and cancer-free controls. The area under the ROC curve (AUC) was calculated and used to evaluate the diagnostic usefulness of TPO and AOX1.

\section{Prognostic analysis}

The prognostic importance of TPO and $A O X 1$ expression was evaluated using the Kaplan-Meier plotter (www.kmplot. com), a widely used online database for survival analysis (19). In the database, gene expression data and relapse-free survival (RFS) information were downloaded from GEO, the European Genome-phenome Archive, and The Cancer Genome Atlas. This database is capable of assessing the effects of 54,000 genes (mRNA, miRNA, protein) on survival in 21 cancer types including PTC. The database contains 502 cases of PTC. Briefly, TPO and AOX1 were entered in the database to acquire RFS plots. Hazard ratios with $95 \%$ confidence intervals and log-rank p-values were calculated and displayed on the main plots.

\section{Statistical analysis}

All data and graphs were generated using GraphPad Prism 7.0. An unpaired $t$-test was performed to analyze differences between two groups. $\mathrm{P}<0.05$ indicated statistical significance. All results were expressed as the mean $\pm \mathrm{SD}$.

\section{Results}

\section{Clinical characteristics of patients}

A summary of the clinicopathological characteristics of the patients, including age at diagnosis, gender, and TNM
Table 1 Clinical characteristics of pediatric PTC patients investigated

\begin{tabular}{lcc}
\hline Parameters & Classes & Number of patients (\%) \\
\hline Age of diagnosis & Total & 64 \\
& Mean age & $9.49(3.25-15.95)$ \\
& $0-6$ & $11(17.1)$ \\
& $6-10$ & $23(36.0)$ \\
Gender & $>10$ & $30(46.9)$ \\
& Male & $20(31.3)$ \\
T stage & Female & $44(68.7)$ \\
& T1 & $9(14.1)$ \\
& T2 & $18(28.1)$ \\
Tymph node & T3 & $21(32.8)$ \\
status & Positive & $16(25)$ \\
TNM Stage & I & $52(81.2)$ \\
\hline PTC, & $12(18.8)$ \\
& Negative & $51(79.6)$ \\
& I & $13(20.4)$ \\
\hline
\end{tabular}

PTC, papillary thyroid carcinoma; TNM, tumor staging system based on primary tumor $(\mathrm{T})$, regional lymph nodes $(\mathrm{N})$ and distant metastasis $(\mathrm{M})$.

stage, is presented in Table 1. The patients ranged in age from 4.02 to 15.33 years (mean, 10.06 years), and $46.9 \%$ of the patients were $\geq 10$ years old. The patients included 20 boys $(31.3 \%)$ and 44 girls (68.7\%), giving a male/female ratio of $1: 2.2$. The numbers of lesions classified as T1, T2, T3, and T4 were 9 (14.1\%), 18 (28.1\%), 21 (32.8\%), and $16(25 \%)$, respectively. Regarding regional lymph nodes metastasis, 52 cases $(81.2 \%)$ were positive, and 12 cases $(18.8 \%)$ were negative. Fifty-one patients (79.6\%) were diagnosed with TNM stage I disease, and 13 patients (20.4\%) were diagnosed with stage II disease.

\section{Gene expression of TPO and AOX1}

As presented in the GSE33630, GSE27155, and GSE3678 datasets, both $T P O$ and $A O X 1$ were expressed at significantly lower level in PTC tumors (Figure 1). Additionally, TPO and $A O X 1$ expression was also lower in tumor tissues than in paired normal thyroid tissues (GSE3467, Figure 1D). Although TPO is expressed exclusively in the thyroid, AOX1 concentrations in plasma might be influenced 

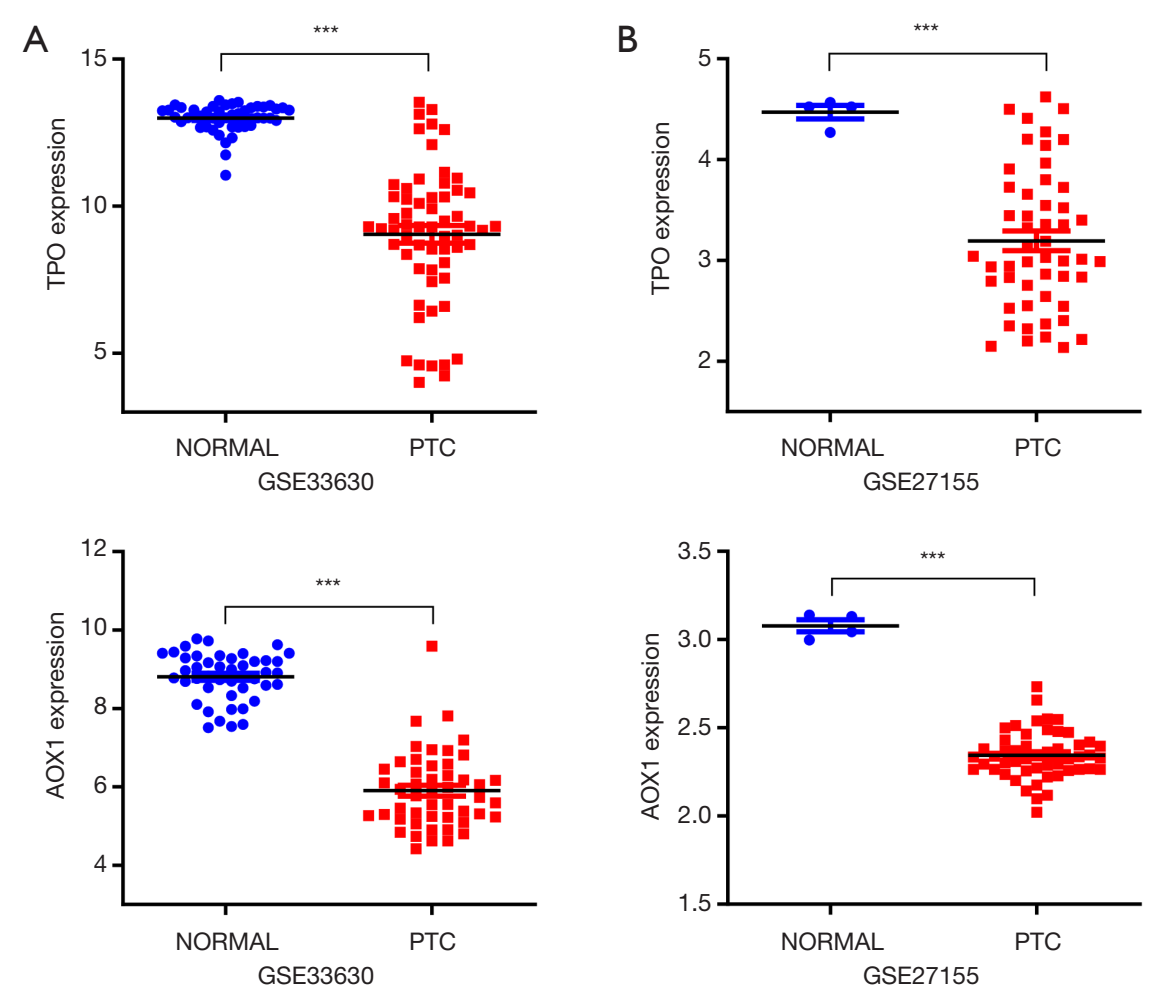

C

D
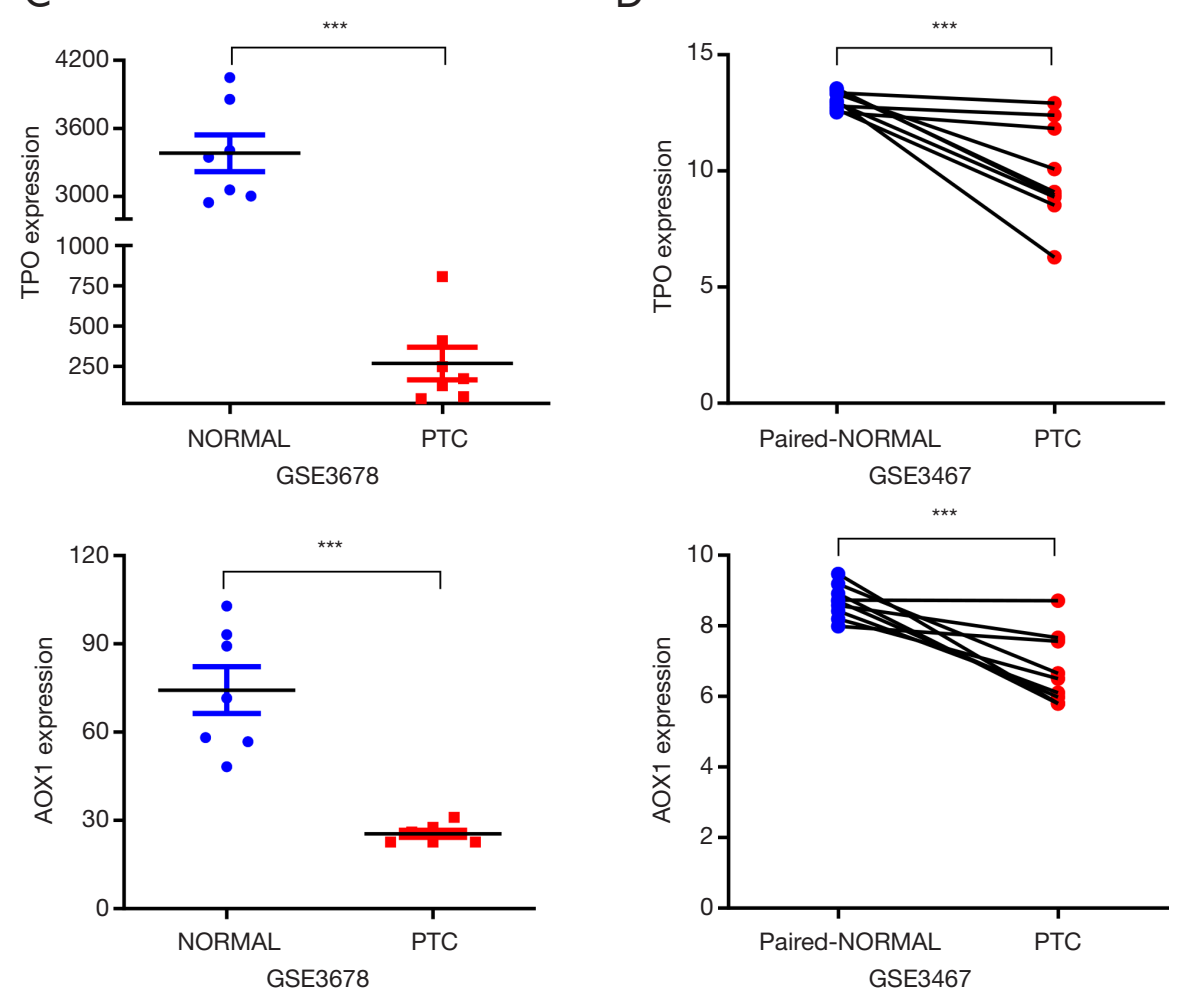

Figure 1 The expression of TPO and AOX1 in different GEO datasets. (A) GSE33630, (B) GSE27155, (C) GSE3678 and (D) GSE3467. Both TPO and AOX1 were significantly decreased in PTC compared with those in normal thyroid. ${ }^{* * *}, \mathrm{P}<0.001$. 

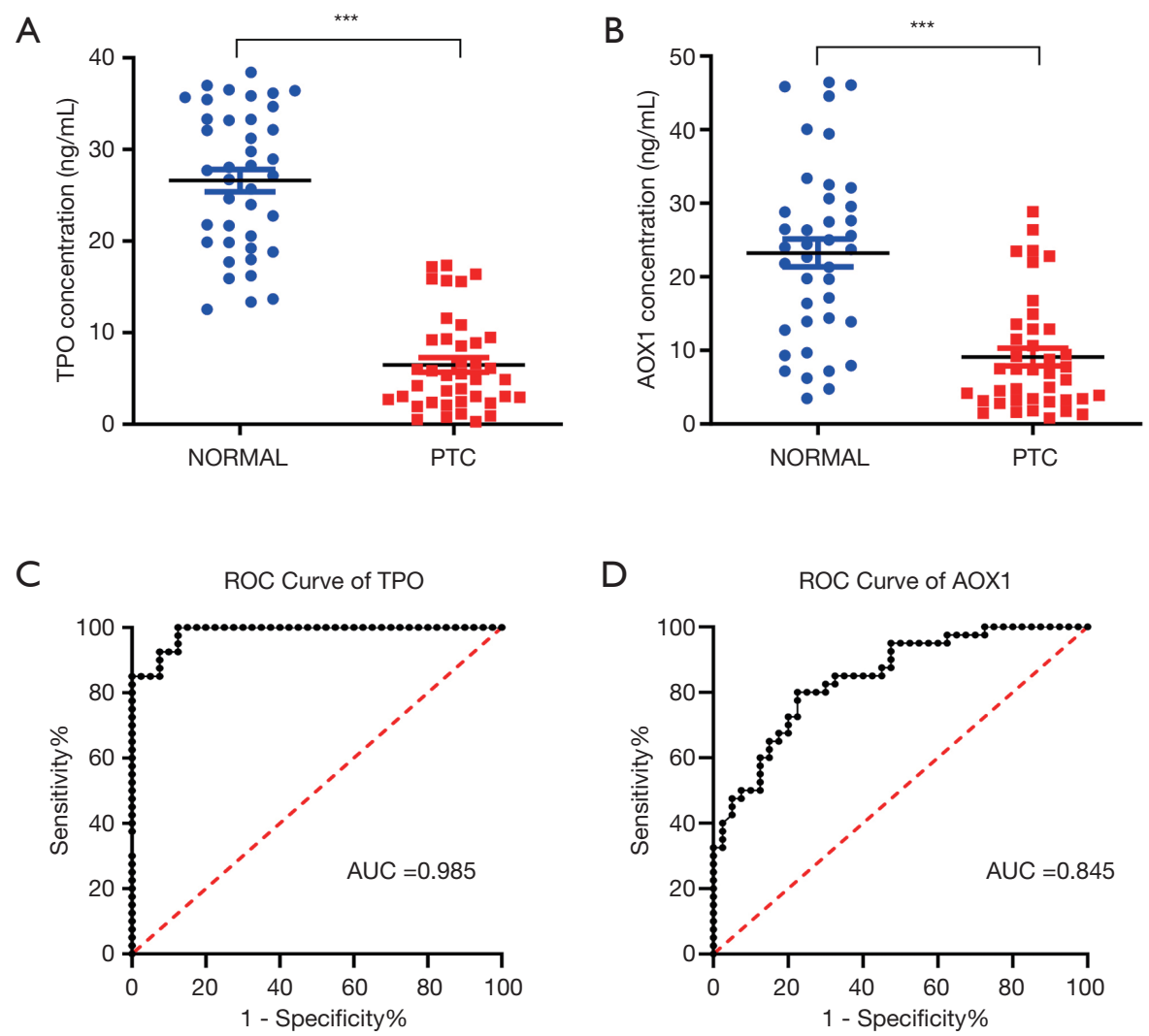

Figure 2 TPO and AOX1 protein content in plasma and ROC curves. (A) TPO protein content and (B) AOX1 content in PTC and cancerfree samples. (C) ROC curve for TPO (AUC =0.985). (D) ROC curve for AOX1 (AUC =0.845). ***, P<0.001.

by other organs or systems. ELISA was further used to explore TPO and AOX1 protein concentrations in plasma. The results illustrated that their plasma levels were significantly lower in children with PTC than in cancer-free controls (Figure 2A,B). These independent datasets and experimental validation confirmed that TPO and $A O X 1$ expression was downregulated at both the gene and protein levels in PTC.

\section{Diagnostic effects of TPO and AOX1}

To assess the predictive accuracy of TPO and AOX1 in plasma, ROC analysis was performed, and AUC was calculated using protein concentrations. The results indicated that TPO had an AUC larger than 0.98 (Figure 2C), and AOX1 had an AUC of 0.845 (Figure 2D). Our ROC analysis indicated that TPO and AOX1 contents in plasma had excellent predictive performance for discriminating PTC from healthy samples.

\section{Prognostic implication of TPO and AOX1}

We further determined the prognostic value of TPO and $A O X 1$, and RFS curves were plotted on www.kmplot.com for patients with PTC $(\mathrm{n}=502)$. As presented in Figure 3, patients with low TPO and $A O X 1$ expression had poorer RFS rates than those with high expression $(\mathrm{P}=0.002$ and $\mathrm{P}=0.038$, respectively). These results suggested that TPO and $A O X 1$ are potential prognostic markers for PTC.

\section{Discussion}

The incidence of pediatric PTC is increasing. To explore its pathogenesis, high-throughput studies have been performed in genetic, epigenetic, and proteomic fields $(14,20,21)$. Various alterations of genes, non-coding RNAs, and proteins have been identified, but it is necessary to verify the reliability of all screened targets in clinical specimens $(22,23)$. In this study, we confirmed that the expression of both TPO and $A O X 1$ was downregulated in PTC tissue, 

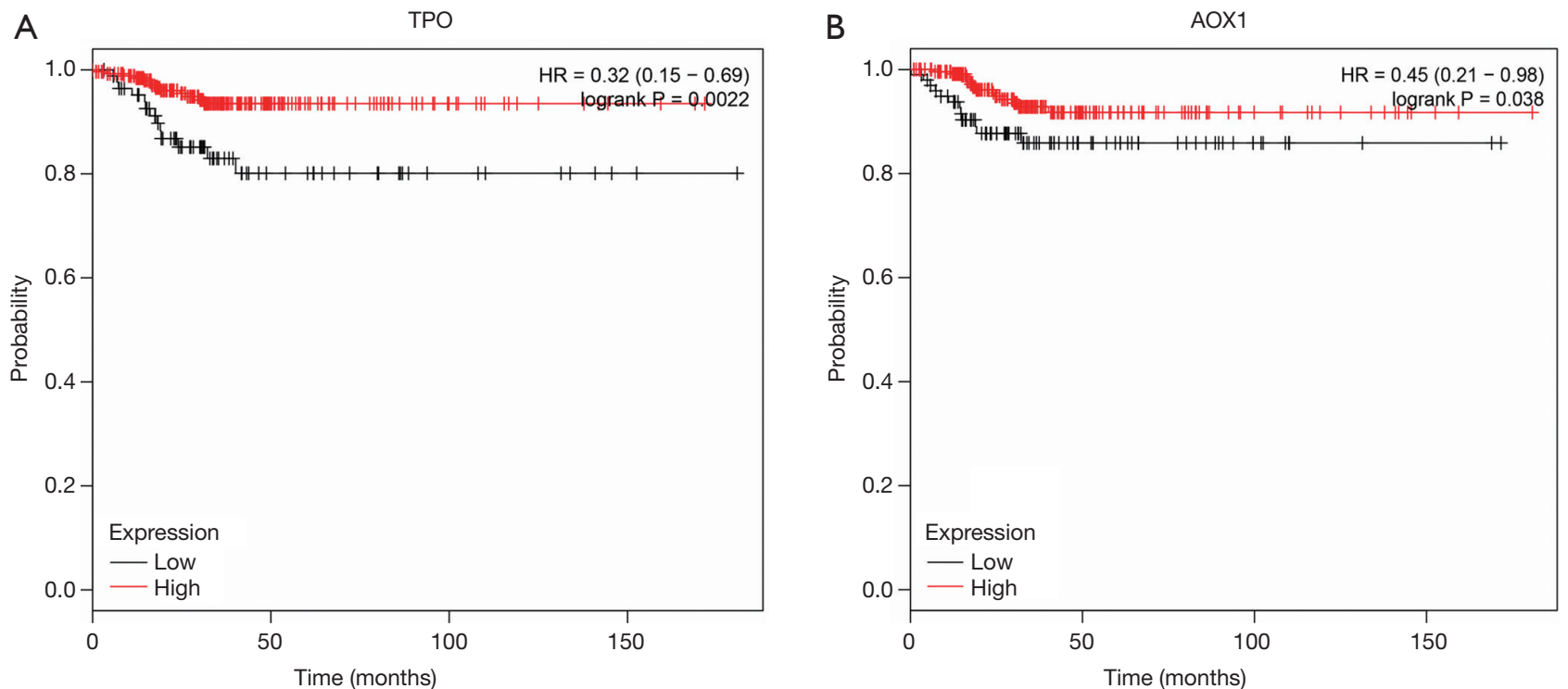

Figure 3 Kaplan-Meier relapse-free survival (RFS) analysis of TPO and AOX1 expression. (A) The survival curves for TPO and (B) AOX1 in low-expression versus high-expression patients.

and their contents in plasma from patients with PTC were decreased. We clarified that TPO and AOX1 might be promising biomarkers for the diagnosis and prognosis of pediatric PTC.

Evidence has illustrated that the incidence of PTC increases with age, and the incidence in adolescents is 10fold higher than that of younger children (24). In our study, $82.9 \%$ of cases of PTC occurred in children $>6$ years old. In accordance with previous literature (25), our study demonstrated that females have a higher incidence, with a female/male ratio of $2.2: 1$, suggesting a female preponderance in PTC. The TNM classification system is the most widely used cancer staging system for most types of cancer. Pediatric patients are classified into stage II if distant metastases are identified; otherwise, all pediatric patients are considered to have stage I disease (26). Compared with the findings in adults, children had larger primary tumors and more lymph node metastases (LNMs) or distant metastases in prior analyses $(25,27)$. In this study, more patients had LNM (81.2\%), and $20.4 \%$ of patients had distant metastasis, indicating that pediatric PTC is more grossly invasive.

TPO expression was significantly downregulated in four independent datasets (GSE33630, GSE27155, GSE3678, and GSE3467), and its protein contents in plasma from patients with PTC were also significantly decreased. Previous research reported that TPO expression was downregulated in PTC tumor tissue $(14,28)$, which is consistent with our results. TPO protein content in plasma was detected for the first time in this study. TPO is an enzyme involved in thyroid hormone synthesis and iodine metabolism that is essential for thyroid function. For thyroid disorders, TPO autoantibody has been detected in $88 \%$ of all patients with various thyroid and autoimmune diseases (29). In thyroid cancer, the expression of TPO is lower in BRAF mutation-positive tumors (30). It is well known that the BRAF (V600E) mutation and RET/PTC rearrangement are common gene alterations in PTC (9). A previous study demonstrated that the BRAF (V600E) mutation, but not RET/PTC rearrangement, is correlated with lower TPO expression (31). In terms of mechanism, TPO downregulation is reportedly driven by constitutive hyperactivation of the MAP kinase signaling pathway mediated by oncogenic BRAF (V600E) (31-33). As a result, $B R A F$ genetic alterations may contribute to the regulation of TPO expression.

$A O X 1$ is a metabolizing enzyme with poorly understood physiological function and substrates. $A O X 1$ has critical roles in xenobiotic metabolism and the regulation of ROS generation (16). In the present study, AOX1 expression in PTC tissue and its content in plasma were significantly reduced. A previous proteomic analysis found that $A O X 1$ was deregulated in pancreatic ductal adenocarcinoma (PDAC), and immunohistochemical analysis verified that 
AOX1 was strongly expressed in approximately $70 \%$ of histologically normal pancreatic cells but absent in PDAC cells (18). Another study demonstrated that reduced $A O X 1$ expression was correlated with tumor stage and metastasis in hepatocellular carcinoma (16). Meanwhile, the function of $A O X 1$ remains controversial in different tumors because $A O X 1$ was reported to promote the proliferation of prostate cancer and colorectal cancer but to inhibit the development of breast cancer (34-36). Mechanically, a molecular study demonstrated that nuclear factor erythroid 2-related factor 2 (Nrf2) binds to $A O X 1$ and strongly activates $A O X 1$ expression (37). Because Kelch-like ECH-associated protein 1 (Keap1)/Nrf2 signaling is a key mediator in thyroid pathophysiology and thyroid cancer, the regulation of $A O X 1$ by the Keap/Nrf2 pathway might account for part of the pathogenesis of PTC (38).

Both gene expression in tissues and protein contents in plasma have promising diagnostic and prognostic potential in many tumors (39-41). A previously study reported that serum TPO was not a useful marker for patients with differentiated thyroid carcinoma (42). However, our ROC analysis indicated that the calculated AUCs for TPO and AOX1 were 0.98 and 0.845 , respectively. Because TPO can improve the diagnosis accuracy in discriminating welldifferentiated thyroid carcinomas from benign tumors, it might be a useful differential diagnosis marker of in PTC $(43,44)$. Moreover, a gene panel containing TPO had good sensitivity $(91 \%)$ and specificity $(81 \%)$ in differentiating PTC from normal thyroid tissue (45). These results revealed that TPO and AOX1 have excellent predictive performance, thus verifying their potential use for clinical diagnosis.

To further explore the prognostic value of TPO and $A O X 1$ in PTC, the correlation between gene expression and patient survival was analyzed using Kaplan-Meier plotter. We found that low expression of TPO and AOX1 may predict poorer survival in PTC. The Kaplan-Meier plotter is a widely used database for assessing the effects of 54,000 genes on survival in 21 cancer types (19). The primary purposes of the tool are the meta-analysis-based discovery and validation of survival biomarkers (46). Many genes have been explored and/or recognized by Kaplan-Meier plotter in several tumor types (47-49). The database contains gene expression data, clinicopathological information, and RFS information for 502 patients with PTC. In a long-term follow-up study, TPO was reported as a prognostic factor for differentiated thyroid cancer (50). Consistent with our results, the expression of $A O X 1$ was downregulated in prostate cancer, and this lower expression portended a poor prognosis (51). The excellent predictive ability in our study suggested that TPO and AOX1 might be potential prognostic markers for patients with PTC.

In conclusion, independent datasets and experimental validation demonstrated that the expression of TPO and $A O X 1$ in tissue and their protein concentrations in blood plasma were significantly reduced in pediatric PTC. Diagnostic analysis revealed that TPO and AOX1 had excellent predictive performance, indicating their potential usefulness for clinical diagnosis. Survival analysis confirmed that TPO and $A O X 1$ can be used as prognostic biomarkers for pediatric PTC, with low expression predicting worse outcomes in patients. This study revealed that TPO and AOX1 might be promising non-invasive biomarkers for PTC diagnosis and prognosis. Additional studies are needed to explore the mechanisms of $T P O$ and $A O X 1$ in pediatric PTC.

\section{Acknowledgments}

We thank Joe Barber Jr., PhD, from Liwen Bianji, Edanz Editing China (www.liwenbianji.cn/ac), for editing the English text of a draft of this manuscript.

Funding: This work was supported by the National Natural Science Foundation of China (No. 81702463, 81702787) and Beijing-Tianjin-Hebei Integration Project (No. J200004).

\section{Footnote}

Reporting Checklist: The authors have completed the REMARK reporting checklist. Available at http://dx.doi. org/10.21037/tp-20-301

Data Sharing Statement: Available at http://dx.doi. org/10.21037/tp-20-301

Conflicts of Interest: All authors have completed the ICMJE uniform disclosure form (available at http://dx.doi. org/10.21037/tp-20-301). The authors have no conflicts of interest to declare.

Ethical Statement: The author is accountable for all aspects of the work in ensuring that questions related to the accuracy or integrity of any part of the work are appropriately investigated and resolved. This study follows the Helsinki Declaration (as revised in 2013) and was approved by the Ethics Committee in Beijing Children's Hospital, Capital 
Medical University (2020-k-168). Informed consent was provided by each patient and/or his/her legal guardians before samples were collected and stored in the biobank.

Open Access Statement: This is an Open Access article distributed in accordance with the Creative Commons Attribution-NonCommercial-NoDerivs 4.0 International License (CC BY-NC-ND 4.0), which permits the noncommercial replication and distribution of the article with the strict proviso that no changes or edits are made and the original work is properly cited (including links to both the formal publication through the relevant DOI and the license). See: https://creativecommons.org/licenses/by-nc-nd/4.0/.

\section{References}

1. Bray F, Ferlay J, Soerjomataram I, et al. Global cancer statistics 2018: GLOBOCAN estimates of incidence and mortality worldwide for 36 cancers in 185 countries. CA Cancer J Clin 2018;68:394-424.

2. Lim H, Devesa SS, Sosa JA, et al. Trends in Thyroid Cancer Incidence and Mortality in the United States, 1974-2013. JAMA 2017;317:1338-48.

3. Schmidt Jensen J, Gronhoj C, Mirian C, et al. Incidence and Survival of Thyroid Cancer in Children, Adolescents, and Young Adults in Denmark: A Nationwide Study from 1980 to 2014. Thyroid 2018;28:1128-33.

4. Du L, Wang Y, Sun X, et al. Thyroid cancer: trends in incidence, mortality and clinical-pathological patterns in Zhejiang Province, Southeast China. BMC Cancer 2018;18:291.

5. La Vecchia C, Malvezzi M, Bosetti C, et al. Thyroid cancer mortality and incidence: a global overview. Int $\mathrm{J}$ Cancer 2015;136:2187-95.

6. Qian ZJ, Jin MC, Meister KD, et al. Pediatric Thyroid Cancer Incidence and Mortality Trends in the United States, 1973-2013. JAMA Otolaryngol Head Neck Surg 2019;145:617-23.

7. Siegel RL, Miller KD, Jemal A. Cancer statistics, 2019. CA Cancer J Clin 2019;69:7-34.

8. Abdullah MI, Junit SM, Ng KL, et al. Papillary Thyroid Cancer: Genetic Alterations and Molecular Biomarker Investigations. Int J Med Sci 2019;16:450-60.

9. Guerra A, Zeppa P, Bifulco M, et al. Concomitant BRAF(V600E) mutation and RET/PTC rearrangement is a frequent occurrence in papillary thyroid carcinoma. Thyroid 2014;24:254-9.

10. Liu $Y$, Gao S, Jin $Y$, et al. Bioinformatics analysis to screen key genes in papillary thyroid carcinoma. Oncol Lett 2020;19:195-204.

11. Kure S, Wada R, Naito Z. Relationship between genetic alterations and clinicopathological characteristics of papillary thyroid carcinoma. Med Mol Morphol 2019;52:181-6.

12. Nagasaka A, Hidaka H, Ishizuki Y. Studies on human iodide peroxidase: its activity in various thyroid disorders. Clin Chim Acta 1975;62:1-4.

13. Cipollini M, Pastor S, Gemignani F, et al. TPO genetic variants and risk of differentiated thyroid carcinoma in two European populations. Int J Cancer 2013;133:2843-51.

14. Li WB, Zhou J, Xu L, et al. Identification of Genes Associated with Papillary Thyroid Carcinoma (PTC) for Diagnosis by Integrated Analysis. Horm Metab Res 2016;48:226-31.

15. Liu Z, Yu P, Xiong Y, et al. Significance of CK19, TPO, and HBME-1 expression for diagnosis of papillary thyroid carcinoma. Int J Clin Exp Med 2015;8:4369-74.

16. Sigruener A, Buechler C, Orso E, et al. Human aldehyde oxidase 1 interacts with ATP-binding cassette transporter-1 and modulates its activity in hepatocytes. Horm Metab Res 2007;39:781-9.

17. Weigert J, Neumeier M, Bauer S, et al. Small-interference RNA-mediated knock-down of aldehyde oxidase 1 in 3T3L1 cells impairs adipogenesis and adiponectin release. FEBS Lett 2008;582:2965-72.

18. Crnogorac-Jurcevic T, Gangeswaran R, Bhakta V, et al. Proteomic analysis of chronic pancreatitis and pancreatic adenocarcinoma. Gastroenterology 2005;129:1454-63.

19. Nagy Á, Lánczky A, Menyhárt O, et al. Validation of miRNA prognostic power in hepatocellular carcinoma using expression data of independent datasets. Sci Rep 2018;8:9227.

20. Navas-Carrillo D, Rodriguez JM, Montoro-Garcia S, et al. High-resolution proteomics and metabolomics in thyroid cancer: Deciphering novel biomarkers. Crit Rev Clin Lab Sci 2017;54:446-57.

21. White MG, Nagar S, Aschebrook-Kilfoy B, et al. Epigenetic Alterations and Canonical Pathway Disruption in Papillary Thyroid Cancer: A Genome-wide Methylation Analysis. Ann Surg Oncol 2016;23:2302-9.

22. Luzón-Toro B, Fernández RM, Martos-Martínez JM, et al. LncRNA LUCAT1 as a novel prognostic biomarker for patients with papillary thyroid cancer. Sci Rep 2019;9:14374.

23. Hao RT, Zheng C, Wu CY, et al. NECTIN4 promotes papillary thyroid cancer cell proliferation, migration, and 
invasion and triggers EMT by activating AKT. Cancer Manag Res 2019;11:2565-78.

24. Hogan AR, Zhuge Y, Perez EA, et al. Pediatric thyroid carcinoma: incidence and outcomes in 1753 patients. J Surg Res 2009;156:167-72.

25. Pan JJ, Zhao L, Cheng R, et al. Thyroid carcinoma in children and adolescents: Clinical characteristics and follow-up from two centers. J Cancer Res Ther 2017;13:715-9.

26. Francis GL, Waguespack SG, Bauer AJ, et al. Management Guidelines for Children with Thyroid Nodules and Differentiated Thyroid Cancer. Thyroid 2015;25:716-59.

27. Hay ID, Johnson TR, Kaggal S, et al. Papillary Thyroid Carcinoma (PTC) in Children and Adults: Comparison of Initial Presentation and Long-Term Postoperative Outcome in 4432 Patients Consecutively Treated at the Mayo Clinic During Eight Decades (1936-2015). World J Surg 2018;42:329-42.

28. Makhlouf AM, Chitikova Z, Pusztaszeri M, et al. Identification of CHEK1, SLC26A4, c-KIT, TPO and TG as new biomarkers for human follicular thyroid carcinoma. Oncotarget 2016;7:45776-88.

29. Doullay F, Ruf J, Codaccioni JL, et al. Prevalence of autoantibodies to thyroperoxidase in patients with various thyroid and autoimmune diseases. Autoimmunity 1991;9:237-44.

30. Bastos AU, Oler G, Nozima BH, et al. BRAF V600E and decreased NIS and TPO expression are associated with aggressiveness of a subgroup of papillary thyroid microcarcinoma. Eur J Endocrinol 2015;173:525-40.

31. Romei C, Ciampi R, Faviana P, et al. BRAFV600E mutation, but not RET/PTC rearrangements, is correlated with a lower expression of both thyroperoxidase and sodium iodide symporter genes in papillary thyroid cancer. Endocr Relat Cancer 2008;15:511-20.

32. Durante C, Puxeddu E, Ferretti E, et al. BRAF mutations in papillary thyroid carcinomas inhibit genes involved in iodine metabolism. J Clin Endocrinol Metab 2007;92:2840-3.

33. Godlewska M, Banga PJ. Thyroid peroxidase as a dual active site enzyme: Focus on biosynthesis, hormonogenesis and thyroid disorders of autoimmunity and cancer. Biochimie 2019;160:34-45.

34. Li W, Middha M, Bicak M, et al. Genome-wide Scan Identifies Role for AOX1 in Prostate Cancer Survival. Eur Urol 2018;74:710-9.

35. Singh B, Shoulson R, Chatterjee A, et al. Resveratrol inhibits estrogen-induced breast carcinogenesis through induction of NRF2-mediated protective pathways. Carcinogenesis 2014;35:1872-80.

36. Zhang W, Chai $W$, Zhu Z, et al. Aldehyde oxidase 1 promoted the occurrence and development of colorectal cancer by up-regulation of expression of CD133. Int Immunopharmacol 2020;85:106618.

37. Fu C, Di L, Han X, et al. Aldehyde oxidase 1 (AOX1) in human liver cytosols: quantitative characterization of AOX1 expression level and activity relationship. Drug Metab Dispos 2013;41:1797-804.

38. Renaud CO, Ziros PG, Chartoumpekis DV, et al. Keap1/ Nrf2 Signaling: A New Player in Thyroid Pathophysiology and Thyroid Cancer. Front Endocrinol (Lausanne) 2019;10:510.

39. Li L, Cai S, Liu S, et al. Bioinformatics analysis to screen the key prognostic genes in ovarian cancer. J Ovarian Res 2017;10:27.

40. Shou X, Li Y, Hu W, et al. Six-gene Assay as a new biomarker in the blood of patients with colorectal cancer: establishment and clinical validation. Mol Oncol 2019;13:781-91.

41. Chen K, Cai Y, Zhang M, et al. Both serum and tissue Galectin-1 levels are associated with adverse clinical features in neuroblastoma. Pediatr Blood Cancer 2018;65:e27229.

42. Ozata M, Bayhan H, Bingol N, et al. Sequential changes in serum thyroid peroxidase following radioiodine therapy of patients with differentiated thyroid carcinoma. J Clin Endocrinol Metab 1995;80:3634-8.

43. Paunovic I, Isic T, Havelka $M$, et al. Combined immunohistochemistry for thyroid peroxidase, galectin-3, CK19 and HBME-1 in differential diagnosis of thyroid tumors. APMIS 2012;120:368-79.

44. Kalfert D, Ludvikova M, Kholova I, et al. Combined use of galectin-3 and thyroid peroxidase improves the differential diagnosis of thyroid tumors. Neoplasma 2020;67:164-70.

45. Stephen JK, Chen KM, Merritt J, et al. Methylation markers differentiate thyroid cancer from benign nodules. J Endocrinol Invest 2018;41:163-70.

46. Győrffy B, Benke Z, Lánczky A, et al. RecurrenceOnline: an online analysis tool to determine breast cancer recurrence and hormone receptor status using microarray data. Breast Cancer Res Treat 2012;132:1025-34.

47. Zhong X, Kan A, Zhang W, et al. CBX3/HP1gamma promotes tumor proliferation and predicts poor survival in hepatocellular carcinoma. Aging (Albany NY) 2019;11:5483-97.

48. Huang N, Wu Z, Hong H, et al. Overexpression of CKS2 
is associated with a poor prognosis and promotes cell proliferation and invasion in breast cancer. Mol Med Rep 2019;19:4761-9.

49. Tu J, Chen J, He M, et al. Bioinformatics analysis of molecular genetic targets and key pathways for hepatocellular carcinoma. Onco Targets Ther 2019;12:5153-62.

Cite this article as: $\mathrm{Yu} \mathrm{Y}$, Wang S, Zhang X, Xu S, Li Y, Liu Q, Yang Y, Sun N, Liu Y, Zhang J, Guo Y, Ni X. Clinical implications of TPO and AOX1 in pediatric papillary thyroid carcinoma. Transl Pediatr 2021;10(4):723-732. doi: 10.21037/ tp-20-301
50. Caballero Y, Lopez-Tomassetti EM, Favre J, et al. The value of thyroperoxidase as a prognostic factor for differentiated thyroid cancer -- a long-term follow-up study. Thyroid Res 2015;8:12.

51. Varisli L. Identification of new genes downregulated in prostate cancer and investigation of their effects on prognosis. Genet Test Mol Biomarkers 2013;17:562-6. 